\title{
A REMARK ON CONDUCTOR, DEPTH AND PRINCIPAL CONGRUENCE SUBGROUPS
}

\author{
MICHITAKA MIYAUCHI AND TAKUYA YAMAUCHI
}

\begin{abstract}
In this paper we study a relation between conductor, depth, and the level of principal congruence subgroups for irreducible admissible representations of $\mathrm{GL}_{n}(F)$ for a non-archimedean local field $F$ of characteristic zero. As a global application, we estimate conductors of unitary irreducible automorphic cuspidal representations of $\mathrm{GL}_{n}\left(\mathbb{A}_{\mathbb{Q}}\right)$ in terms of principal congruence subgroups. We also give an explicit formula for the dimension of fixed vectors with respect to principal congruence subgroups for irreducible admissible representations of $\mathrm{GL}_{2}(F)$ as a local application.
\end{abstract}

\section{Contents}

1. Introduction

2. Essentially square integrable representations

3. Parabolically induced representations

4. Applications

4.1. Lower and upper bounds of the conductor

4.2. The number of $K(m)$-fixed vectors when $n=2$

References

Date: February 22, 2022.

Key words and phrases. depth, conductor, principal congruence subgroups.

The first author is partially supported by JSPS KAKENHI Grant Number (B) No.19H01778. The second author is partially supported by JSPS KAKENHI Grant Number (B) No.19H01778. 


\section{INTRODUCTION}

Let $F$ be a non-archimedean local field of characteristic zero and $\mathcal{O}=\mathcal{O}_{F}$ the ring of integers of $F$. Let $\mathfrak{p}=\varpi \mathcal{O}$ be the maximal ideal of $\mathcal{O}$ where $\varpi$ is a fixed uniformizer of $F$. Put $\mathbb{F}=\mathcal{O} / \mathfrak{p}$. For an irreducible generic admissible representation $\pi$ of $\mathrm{GL}_{n}(F)$, there are several important invariants characterizing $\pi$. In this paper, we focus on the conductor $c(\pi)$ of $\pi$ defined in [8] (there is a gap in the proof there but later it is modified in [7], [11]) and the depth $\rho(\pi)$ defined in [12]. Both of invariants are defined by using families of suitable open compact subgroups of $\mathrm{GL}_{n}(F)$. Therefore, it is quite natural to ask any substantial proportion of the principal congruence subgroups among such families.

In this paper, we study a condition when $\pi$ has a non-trivial fixed vector by a principal congruence subgroup in terms of the conductor $c(\pi)$ through study of the depth $\rho(\pi)$. Let us fix some notation to explain our results. Put $K=\mathrm{GL}_{n}(\mathcal{O})$. For each positive integer $m$, let $K_{1}(m)$ be the subgroup of $K$ consisting of any element $g$ whose reduction modulo $\mathfrak{p}$ satisfies that the $n$-th row of $g \bmod \mathfrak{p}^{m}$ is $(0, \ldots, 0,1)$. We also define the principal congruence subgroup $K(m)$ of level $m$ to be the kernel of the mod $\mathfrak{p}^{m}$-reduction $K \longrightarrow \mathrm{GL}_{n}\left(\mathcal{O} / \mathfrak{p}^{m}\right)$. The conductor $c(\pi)$ of $\pi$ is defined as the minimal non-negative integer $m$ of which $\pi$ has a non-trivial $K_{1}(m)$-fixed vector. Then we will prove the following:

Theorem 1.1. Let $\pi$ be an irreducible essentially square integrable representation of $\mathrm{GL}_{n}(F)$ and $m$ a non-negative integer. The following two conditions are equivalent:

(1) $\pi$ has a non-trivial $K(m)$-fixed vector;

(2) $c(\pi) \leq m n$.

As is known, any irreducible generic representation $\pi$ can be written by

$$
\pi=\operatorname{Ind}_{P_{\underline{n}}(F)}^{\mathrm{GL}_{n}(F)}\left(\tau_{1} \otimes \cdots \otimes \tau_{k}\right)
$$

where $P_{\underline{n}}$ is the standard parabolic subgroup associated to the partition $\underline{n}=\left(n_{1}, \ldots, n_{k}\right)$ of $n$ such that its Levi subgroup is isomorphic to $\mathrm{GL}_{n_{1}} \times \cdots \times \mathrm{GL}_{n_{k}}$ (cf. Theorem 9.3 of [13]). Further each $\tau_{i}(1 \leq i \leq k)$ is an irreducible essentially square integrable representation of $\mathrm{GL}_{n_{i}}(F)$. Then we will show the following: 
Theorem 1.2. Let $\pi$ be as in (1.1) and $m$ a non-negative integer. The following two conditions are equivalent:

(1) $\pi$ has a non-trivial $K(m)$-fixed vector;

(2) $c\left(\tau_{i}\right) \leq m n_{i}$ for each $1 \leq i \leq k$.

It follows immediately from Theorem 1.1 and Theorem 1.2 that

Corollary 1.3. Let $\pi$ be an irreducible generic representation of $\mathrm{GL}_{n}(F)$ and $m$ a nonnegative integer. The following two conditions are equivalent:

(1) $\pi$ has a non-trivial $K(m)$-fixed vector but $\pi^{K(m-1)}=0$;

(2) $m<c(\pi) \leq m n$.

where $\pi^{K(m-1)}=0$ means no condition when $m=0$.

Further, if $\pi$ is essentially square integrable, then the condition $m<c(\pi) \leq m n$ is replaced with $(m-1) n<c(\pi) \leq m n$.

A motivation in our study comes up to authors in an estimation of upper and lower bounds of the conductor of automorphic families in the setting of low lying zeros [16], [9] (see also Remark 4.2).

This paper is organized as follows. In Section 2, we give a proof of Theorem 1.1 which is a balk of this paper and the general case follows through the discussion in Section 3. Finally, we give some applications in both of global and local situation. The global one is about an estimation of the global conductor of a cuspidal representation of $\mathrm{GL}_{n}\left(\mathbb{A}_{\mathbb{Q}}\right)$. Finally, the dimension of $K(m)$-fixed vectors in the case when $n=2$ will be discussed.

Acknowledgments. We would like to thank professors H-H. Kim and S. Wakatsuki for inspiring the authors to address this problem. We thank the referee for helping us to correct and improve our paper greatly.

\section{EsSENTIALly SQUARE INTEGRABLE REPRESENTATIONS}

Let $\pi$ be an irreducible admissible representation of $\mathrm{GL}_{n}(F)$. The depth $\rho(\pi)$ of $\pi$ is defined in [12]. Due to [2], we may use the lattice-theoretic realization of unrefined minimal $K$-types in $\left[3\right.$. Let $\mathfrak{A}$ be a hereditary order in $M_{n}(F)$. We denote by $\mathfrak{P}$ the 
Jacobson radical of $\mathfrak{A}$. There exists a positive integer $e(\mathfrak{A})$ which satisfies $\mathfrak{P}^{e(\mathfrak{A})}=\varpi \mathfrak{A}$. By [12] Theorem 5.2, the depth $\rho(\pi)$ of $\pi$ is equal to the least $i / e(\mathfrak{A})$ such that $\pi$ has a non-zero $1+\mathfrak{P}^{i+1}$-fixed vector for a non-negative integer $i$.

Proposition 2.1. Let $\pi$ be an irreducible essentially square integrable representation of $\mathrm{GL}_{n}(F)$. For any positive integer $m$, the following two conditions are equivalent:

(i) $\rho(\pi) \leq m-1$;

(ii) $\pi$ has a non-zero $K(m)$-fixed vector.

Proof. (i) $\Longrightarrow$ (ii). Let $\mathfrak{A}$ be a hereditary order in $M_{n}(F)$ so that $\pi$ has a non-zero $1+\mathfrak{P}^{i+1}$ fixed vector and $\rho(\pi)=i / e(\mathfrak{A})$. Replacing by a conjugate if necessary, we may assume that $M_{n}(\mathcal{O}) \supset \mathfrak{A} \supset \mathfrak{P} \supset \varpi M_{n}(\mathcal{O})$. Suppose that $\rho(\pi)=i / e(\mathfrak{A}) \leq m-1$. Then we have

$$
1+\mathfrak{P}^{i+1} \supset 1+\mathfrak{P}^{e(\mathfrak{A})(m-1)+1}=1+\varpi^{m-1} \mathfrak{P} \supset 1+\varpi^{m} M_{n}(\mathcal{O})=K(m) .
$$

So $\pi$ contains a non-zero $K(m)$-fixed vector.

(ii) $\Longrightarrow\left(\right.$ i). Suppose that $\pi$ has a non-zero $K(m)$-fixed vector. Set $\mathfrak{A}=M_{n}(\mathcal{O})$. Then $\mathfrak{A}$ is a hereditary order in $M_{n}(F)$ with $e(\mathfrak{A})=1$. Since $K(m)=1+\varpi^{m} M_{n}(\mathcal{O})=1+\mathfrak{P}^{m}$, the depth $\rho(\pi)$ of $\pi$ is less than or equal to $(m-1) / e(\mathfrak{A})=m-1$.

Proposition 2.2. Let $\pi$ be an irreducible essentially square integrable representation of $\mathrm{GL}_{n}(F)$. For any positive integer $m$, the following two conditions are equivalent:

(i) $\rho(\pi) \leq m-1$;

(ii) $c(\pi) \leq m n$.

Proof. By [10] Theorem 3.1, we have $\rho(\pi)=\max \left\{\frac{c(\pi)-n}{n}, 0\right\}$. Suppose that $\rho(\pi)=0$. Then we have $c(\pi) \leq n$. In this case, both conditions (i) and (ii) hold for any positive integers $m$. If $\rho(\pi)=\frac{c(\pi)-n}{n}$, then we see that

$$
\rho(\pi)=\frac{c(\pi)-n}{n} \leq m-1 \Longleftrightarrow c(\pi)-n \leq m n-n \Longleftrightarrow c(\pi) \leq m n .
$$

This completes the proof.

We shall prove Theorem 1.1 . 
Proof of Theorem 1.1. If $m$ is a positive integer, then the conditions (1) and (2) are equivalent by Propositions 2.1 and 2.2. Suppose that $m=0$. Then $c(\pi) \leq m n=0$ if and only if $c(\pi)=0$. We note that $K_{1}(0)=K(0)=\mathrm{GL}_{n}(\mathcal{O})$. By the definition of $c(\pi)$, we see that $c(\pi)=0$ if any only if $\pi$ has a non-zero $K(0)$-fixed vector. This completes the proof.

\section{Parabolically induced Representations}

Let $\underline{n}=\left(n_{1}, \ldots, n_{k}\right)$ be a partition of $n$. We denote by $P_{\underline{n}}$ the standard parabolic subgroup of $\operatorname{GL}_{n}(F)$ associated to $\underline{n}$. Then $P_{\underline{n}}$ is the group consisting of all the elements $p$ in $\mathrm{GL}_{n}(F)$ which have the form

$$
p=\left(\begin{array}{cccc}
p_{11} & p_{12} & \cdots & p_{1 k} \\
& p_{22} & \cdots & p_{2 k} \\
& & \ddots & \vdots \\
& & & p_{k k}
\end{array}\right),
$$

where $p_{i j} \in M_{n_{i}, n_{j}}(F)$. The Levi subgroup $M$ of $P_{\underline{n}}$ is the group of the block diagonal matrices in $P_{\underline{n}}$, and isomorphic to $\mathrm{GL}_{n_{1}}(F) \times \cdots \times \mathrm{GL}_{n_{k}}(F)$. For any non-negative integer $m$, we define an open compact subgroup $K(m)_{n_{i}}$ of $\mathrm{GL}_{n_{i}}(F)$ by

$$
K(0)_{n_{i}}=\mathrm{GL}_{n_{i}}(\mathcal{O}), K(m)_{n_{i}}=1+\varpi^{m} M_{n_{i}}(\mathcal{O}), m \geq 1
$$

For a representation $\pi$ of a group $G$ and a subgroup $H$ of $G$, we denote by $\pi^{H}$ the space of $H$-fixed vectors in $\pi$.

Lemma 3.1. Let $\pi=\operatorname{Ind}_{P_{\underline{n}}(F)}^{\mathrm{GL}_{n}(F)}\left(\tau_{1} \otimes \cdots \otimes \tau_{k}\right)$ be the parabolically induced representation where $\tau_{i}$ is a smooth representation of $\mathrm{GL}_{n_{i}}(F)$, for $1 \leq i \leq k$. Then $\pi$ has a non-trivial $K(m)$-fixed vector if and only if $\tau_{i}^{K(m)_{n_{i}}} \neq\{0\}$ for all $1 \leq i \leq k$. It also holds that

$$
\operatorname{dim} \pi^{K(m)}=\left|P_{\underline{n}} \backslash \mathrm{GL}_{n}(F) / K(m)\right| \prod_{i=1}^{k} \operatorname{dim} \tau_{i}^{K(m)_{n_{i}}}, m \geq 0 .
$$

Proof. Let $\Omega$ be a complete system of representatives for $P_{\underline{n}} \backslash \mathrm{GL}_{n}(F) / K(m)$. Set $\tau=$ $\tau_{1} \otimes \cdots \otimes \tau_{k}$. By [1] Lemma 2.24, there is an isomorphism of complex vector spaces

$$
\pi^{K(m)} \simeq\left\{f: \Omega \rightarrow \tau \mid f(g) \in \tau^{M \cap g K(m) g^{-1}}, g \in \Omega\right\} .
$$


By the Iwasawa decomposition $\mathrm{GL}_{n}(F)=P_{\underline{n}} K(0)$, we may take $\Omega$ so that $\Omega \subset K(0)$. Since $K(0)$ normalizes $K(m)$, we have

$$
\pi^{K(m)} \simeq\left\{f: \Omega \rightarrow \tau \mid f(g) \in \tau^{M \cap K(m)}, g \in \Omega\right\}
$$

So the claim follows because

$$
M \cap K(m)=K(m)_{n_{1}} \times \cdots \times K(m)_{n_{k}}
$$

and

$$
\tau^{M \cap K(m)}=\tau_{1}^{K(m)_{n_{1}}} \otimes \cdots \otimes \tau_{k}^{K(m)_{n_{k}}} .
$$

This completes the proof.

We shall prove Theorem 1.2 .

Proof of Theorem 1.2. By Lemma 3.1, $\pi$ has a non-trivial $K(m)$-fixed vector if and only if $\tau_{i}^{K(m)_{n_{i}}} \neq\{0\}$ for all $1 \leq i \leq k$. Since $\tau_{i}$ is irreducible and essentially square integrable, it follows from Theorem 1.1 that the latter condition is equivalent to $c\left(\tau_{i}\right) \leq m n_{i}$ for each $1 \leq i \leq k$.

\section{Applications}

In this section, we give some applications of the previous results. First we give both of lower and upper bounds of the conductor for each automorphic cuspidal representation $\Pi$ of $\mathrm{GL}_{n}\left(\mathbb{A}_{\mathbb{Q}}\right)$ when $\Pi$ has a fixed vector for some principal congruence subgroup. Next, we estimate the number of $K(m)$-fixed vectors for any irreducible admissible representation of $\mathrm{GL}_{2}(F)$. When the representation in question has minimal conductor among twists, the result is well known due to Casselman [5] and Tunnell [17]. However, here we do not assume this minimality condition on the representations.

We should mention that emeritus professor Hiroyuki Yoshida at Kyoto university wrote a Japanese book entitled as the theory of automorphic forms. In Section 5, p.255 of Chapter IX, he mentioned that he gave several lectures in Paris around 1982 and the contents are based on his lecture notes prepared by himself. They are closely related to our contents below (Section 4.2) but not a copy of them. Further, he also assumed the representations have the minimal conductors as in the case of Casselman-Tunnell. The 
authors in this article hope his book would be published in English in near future. We should also mention that our method relies on techniques from Casselman-Tunnell while Yoshida took a down-to-earth (but beautiful, educative) method.

4.1. Lower and upper bounds of the conductor. Let us work on the ring of adeles $\mathbb{A}_{\mathbb{Q}}$ of $\mathbb{Q}$ only for simplicity. A similar result for any number field would be quite easy to formulate, but it is cumbersome and therefore omitted. Let $\Pi=\otimes_{p}^{\prime} \Pi_{p}$ be a unitary irreducible automorphic cuspidal representation of $\mathrm{GL}_{n}\left(\mathbb{A}_{\mathbb{Q}}\right)$. By Corollary in p.190 of [15], at each prime $p$, the local component $\Pi_{p}$ is generic. For each positive integer $N$, put $K(N):=\operatorname{Ker}\left(\mathrm{GL}_{n}(\widehat{\mathbb{Z}}) \stackrel{\bmod }{\longrightarrow} \mathrm{GL}_{n}(\widehat{\mathbb{Z}})\right)$. We denote by $c(\Pi)$ the global conductor of $\Pi$ which is defined by $c(\Pi)=\prod_{p<\infty} p^{c\left(\Pi_{p}\right)}$.

Theorem 4.1. Let $\Pi=\otimes_{p}^{\prime} \Pi_{p}$ be a unitary irreducible automorphic cuspidal representation of $\mathrm{GL}_{n}\left(\mathbb{A}_{\mathbb{Q}}\right)$. Suppose that there exists a positive integer $N$ such that $\Pi^{K(N)} \neq 0$ but $\Pi^{K(d)}=0$ for any proper divisor $d$ of $N$. Then, it holds that

$$
\max \left\{\prod_{p \mid N} p, N \cdot \prod_{p \mid N} p^{-1}\right\} \leq c(\Pi) \leq N^{n} .
$$

Proof. It suffice to consider each prime $p$ dividing $N$. Put $e_{p}:=\operatorname{ord}_{p}(N)$. By Theorem 1.2 we have $c\left(\Pi_{p}\right) \leq e_{p} n$. The upper bound follows from this. For the lower bound in the claim, we write $\Pi_{p}=\operatorname{Ind}_{P_{\left(n_{1}, \ldots, n_{k}\right)}\left(\mathbb{Q}_{p}\right)}^{\mathrm{GL}_{n}\left(\mathbb{Q}_{p}\right)}\left(\tau_{1} \otimes \cdots \otimes \tau_{k}\right)$ as explained in right after Theorem 1.1. By Theorem 1.2 and the assumption, there exists $1 \leq i \leq k$ such that $c\left(\tau_{i}\right)>\left(e_{p}-1\right) n_{i} \geq e_{p}-1$. Hence $c\left(\Pi_{p}\right) \geq e_{p}-1$. Clearly, $c\left(\Pi_{p}\right) \geq 1$ if $p \mid N$ by assumption. The claim follows from this.

Remark 4.2. Let $H$ be a reductive group over $\mathbb{Q}$ with a homomoprhism $r: H \longrightarrow \mathrm{GL}_{n}$. In several settings, automorphic cuspidal representations on $H\left(\mathbb{A}_{\mathbb{Q}}\right)$ are transferred to automorphic representations on $\mathrm{GL}_{n}\left(\mathbb{A}_{\mathbb{Q}}\right)$. A basic and important example is the Arthur's endoscopic classification of automorphic representations for classical groups. To apply this kind of results to, for example, equidistribution theorems as in [16], [9] in the level aspects, we need to know an explicit form of the fundamental lemma for Hecke elements. In most cases, it is done for the characteristic functions for principal congruence subgroups. 
Therefore, it is quite natural to ask any useful relations between various known invariants, as conductors or depths, of automorphic representations and the existence of fixed vectors in some principal congruence subgroups. Our study suggests to consider new forms with respect to principal congruence subgroups introduced in Definition 5.1 in [9]. This might be helpful to guarantee Hypothesis 11.4, p.129 of [16].

4.2. The number of $K(m)$-fixed vectors when $n=2$. To ask if a representation has a non-zero $K(0)$-fixed vector is easy. Therefore, we suppose $m$ is positive. For any quasicharacter $\lambda: F^{\times} \longrightarrow \mathbb{C}^{\times}$and a non-negative integer $r$, let $\delta(c(\lambda) \leq r)=1$ if the inequality $c(\lambda) \leq r$ holds and $\delta(c(\lambda) \leq r)=0$ otherwise.

For non-supercuspidal representations of $\mathrm{GL}_{2}(F)$, we have the following

Proposition 4.3. Let $r$ be a positive integer. It holds that

(1) if $\pi=\pi\left(\chi_{1}, \chi_{2}\right)$ be a principal series representation with quasi-characters $\chi_{i}$ : $F^{\times} \longrightarrow \mathbb{C}^{\times}$for $i=1,2$, then $\operatorname{dim} \pi^{K(r)}=q^{r-1}(q+1) \delta\left(c\left(\chi_{1}\right) \leq r\right) \delta\left(c\left(\chi_{2}\right) \leq r\right) ;$

(2) if $\pi=\chi \otimes$ St be the twisted Steinberg representation by a quasi-character $\chi$ : $F^{\times} \longrightarrow \mathbb{C}^{\times}$, then $\operatorname{dim} \pi^{K(r)}=\left(q^{r}+q^{r-1}-1\right) \delta(c(\chi) \leq r)$.

Proof. For the first claim by Lemma 3.1, we have

$$
\operatorname{dim} \pi^{K(r)}=\left|B\left(\mathcal{O} / \varpi^{r} \mathcal{O}\right)\right|^{-1}\left|\mathrm{GL}_{2}\left(\mathcal{O} / \varpi^{r} \mathcal{O}\right)\right| \delta\left(c\left(\chi_{1}\right) \leq r\right) \delta\left(c\left(\chi_{2}\right) \leq r\right)
$$

Then the claim follows from $\left|B\left(\mathcal{O} / \varpi^{r} \mathcal{O}\right)\right|^{-1}\left|\mathrm{GL}_{2}\left(\mathcal{O} / \varpi^{r} \mathcal{O}\right)\right|=q^{r-1}(q+1)$ since $r$ is positive.

For the second claim, recall the exact sequence

$$
0 \longrightarrow \chi \otimes \operatorname{St} \longrightarrow \pi\left(\chi|*|^{\frac{1}{2}}, \chi|*|^{-\frac{1}{2}}\right) \longrightarrow \chi \otimes \operatorname{det} \longrightarrow 0
$$

Taking $K(r)$-fixed parts is an exact functor by Proposition 2.1.7 of [6]. The claim follows from this by using Lemma 3.1 again.

Remark 4.4. For any principal series representation $\pi$ of $\mathrm{GL}_{n}(F)$ which is not necessarily irreducible, by using Lemma 3.1, we would have a similar result as in Proposition 4.3. (1). We left this problem to the interesting readers. 
We shall consider supercuspidal representations of $\mathrm{GL}_{2}(F)$. For two quasi-character $\lambda_{1}, \lambda_{2}: F^{\times} \longrightarrow \mathbb{C}^{\times}$, we write $\lambda_{1} \sim \lambda_{2}$ if $\lambda_{1} \lambda_{2}^{-1}$ is unramified. For each non-negative integer $r$, let $X_{\leq r}\left(\operatorname{resp} . X_{r}\right)$ be the set of all quasi-characters with conductors less than or equal to $r$ (resp. equal to $r$ ). Choose a complete system $L_{\leq r}$ (resp. $L_{r}$ ) of representatives of $X_{\leq r} / \sim\left(\operatorname{resp} . X_{r} / \sim\right)$. Notice that $X_{\leq r} / \sim$ is isomorphic to $\mathcal{O}_{F}^{\times} /\left(1+\varpi^{r} \mathcal{O}_{F}\right)$ via the Pontryagin dual and it follows from this that

$$
\left|L_{0}\right|=1,\left|L_{1}\right|=q-2,\left|L_{i}\right|=(q-1)^{2} q^{i-2}(i \geq 2) .
$$

For each character $\lambda: F^{\times} \longrightarrow \mathbb{C}^{\times}$, we define the function $\xi_{\lambda}^{(m)}$ on $F^{\times}$by

$$
\xi_{\lambda}^{(m)}(x)=\left\{\begin{array}{cl}
\lambda(x) & \text { if } \operatorname{ord}_{\varpi}(x)=-m \\
0 & \text { otherwise }
\end{array}\right.
$$

Notice that for an unramified character $\lambda^{\prime}: F^{\times} \longrightarrow \mathbb{C}^{\times}$, we see easily that $\xi_{\lambda \lambda^{\prime}}^{(m)}=\lambda^{\prime}(\varpi)^{-m} \xi_{\lambda}^{(m)}$.

Lemma 4.5. Let $\psi$ be a non-trivial additive character of $F$ with the conductor $c(\psi)$ (hence, the largest integer which satisfies $\left.\psi\left(\varpi^{-c(\psi)}\right)=1\right)$. Let $\pi$ be an irreducible supercuspidal representation of $\mathrm{GL}_{2}(F)$ and denote by $\mathcal{K}_{\psi}(\pi)$ the Krillov model of $\pi$ with respect to $\psi$. Suppose $r \geq-c(\psi)$. Then $\mathcal{K}_{\psi}(\pi)^{K(r)}$ has a basis $B_{r}$ consisting of all $\xi_{\lambda}^{(m)}, \lambda \in L_{\leq r}$ satisfying $c(\pi \otimes \lambda)+c(\psi)-r \leq m \leq c(\psi)+r$.

Proof. This is well-known for experts but we give a sketch of a proof (see also p.102 of [14]). Since $F^{\times}$is abelian and $F^{\times}=\bigcup_{n \in \mathbb{Z}} \varpi^{n} \mathcal{O}^{\times}$, by using the character expansion, each element $v$ of $\mathcal{K}_{\psi}(\pi)^{K(r)}$ can be written as a finite sum $v=\sum_{\lambda} \sum_{m \in \mathbb{Z}} a_{m, \lambda} \xi_{\lambda}^{(m)}$ where $a_{m, \lambda}=\int_{\varpi^{-m} \mathcal{O} \times} \lambda(x) \pi\left(\left(\begin{array}{ll}x & 0 \\ 0 & 1\end{array}\right)\right) v d x^{\times}$and $\lambda$ runs over a finite subset of $\bigcup_{r \geq 0} L_{r}$. Since $v$ is $K(r)$-fixed, $a_{m, \lambda}=0$ unless $c(\lambda) \leq r$. Since $v$ is fixed by $\left(\begin{array}{cc}1 & \varpi^{r} \\ 0 & 1\end{array}\right)$, it follows from 


$$
\begin{gathered}
\left(\begin{array}{ll}
x & 0 \\
0 & 1
\end{array}\right)\left(\begin{array}{cc}
1 & \varpi^{r} \\
0 & 1
\end{array}\right)\left(\begin{array}{ll}
x & 0 \\
0 & 1
\end{array}\right)^{-1}=\left(\begin{array}{cc}
1 & x \varpi^{r} \\
0 & 1
\end{array}\right) \text { that } \\
\left.a_{m, \lambda}=\int_{\varpi^{-m} \mathcal{O} \times} \lambda(x) \psi\left(x \varpi^{r}\right) \pi\left(\begin{array}{ll}
x & 0 \\
0 & 1
\end{array}\right)\right) v d x^{\times}=\psi\left(\varpi^{r-m}\right) a_{m, \lambda} .
\end{gathered}
$$

Therefore, $a_{m, \lambda}=0$ unless $r-m \geq-c(\psi)$. Let $w=\left(\begin{array}{cc}0 & 1 \\ -1 & 0\end{array}\right)$ be the Weyl element of $\mathrm{GL}_{2}(F)$. By Lemma 2.1 of [14] which is the formula (9) of H. Yoshida [18], we have $\pi(w) \xi_{\lambda}^{(m)}=\varepsilon\left(\pi \otimes \lambda^{-1}\right) \xi_{\omega_{\pi} \lambda^{-1}}^{\left(m_{w}\right)}$ where $\varepsilon\left(\pi \otimes \lambda^{-1}\right)$ is the epsilon factor and $m_{w}=c\left(\pi \otimes \lambda^{-1}\right)+$ $2 c(\psi)-m$. Since $w^{-1}\left(\begin{array}{cc}1 & \varpi^{r} \\ 0 & 1\end{array}\right) w=\left(\begin{array}{cc}1 & 0 \\ -\varpi^{r} & 1\end{array}\right)$, the similar argument shows that $a_{m, \lambda}=0$ unless $r-m_{w} \geq-c(\psi)$. Summing up we have

$$
c(\lambda) \leq r, c\left(\pi \otimes \lambda^{-1}\right)+c(\psi)-r \leq m \leq r+c(\psi) .
$$

This yields $\mathcal{K}_{\psi}(\pi)^{K(r)} \subset B_{r}$.

Conversely, the above argument also shows $\xi_{\lambda}^{(m)}$ is fixed by $K(r)$ if and only if in addition to above two conditions (4.1), $r \geq-c(\psi)$. This completes a proof.

Lemma 4.6. Let $\pi$ be an irreducible supercuspidal representation of $\mathrm{GL}_{2}(F)$ and $r$ a positive integer. If $c(\pi)>2 r$, then $\pi^{K(r)}=0$. Contrary, if $\pi^{K(r)} \neq 0$ (hence $\left.c(\pi) \leq 2 r\right)$, then

$$
\operatorname{dim} \pi^{K(r)}=\sum_{i=0}^{r} \sum_{\lambda \in L_{i}}\left(2 r-c\left(\pi \otimes \lambda^{-1}\right)+1\right)=\sum_{i=0}^{r} \sum_{\lambda \in L_{i}}(2 r-c(\pi \otimes \lambda)+1) .
$$

Proof. The former claim follows from Theorem 1.1 for $n=2$ and $m=r$. The latter formula is given by the counting all elements in the set $B_{r}$ in Lemma 4.5 for an additive character $\psi$ of $F$ with $c(\psi)=0$.

Let $\pi$ is an irreducible supercuspidal representation of $\mathrm{GL}_{2}(F)$. By Theorem 1.1, we have $\operatorname{dim} \pi^{K(m)}=0$ unless $c(\pi) \leq 2 m$. It follows from [10] Proposition 2.2 that $c(\pi)=$ $2 \rho(\pi)+2 \geq 2$. We say that $\pi$ is minimal if

$$
c(\pi) \leq c(\pi \otimes \chi)
$$


for any quasi-character $\chi$ of $F^{\times}$. Suppose that $\pi$ is minimal. Then it follows from [17] Proposition 3.5 that

$$
c(\pi \otimes \chi)=\left\{\begin{array}{cl}
c(\pi) & \text { if } 2 c(\chi) \leq c(\pi) \\
2 c(\chi) & \text { if } c(\pi)<2 c(\chi)
\end{array}\right.
$$

Proposition 4.7. Let $\pi$ be an irreducible minimal supercuspidal representation of $\mathrm{GL}_{2}(F)$. Set $r=\left\lfloor\frac{c(\pi)}{2}\right\rfloor$. Then, for any integer $m$ which satisfies $c(\pi) \leq 2 m$, we have

$$
\operatorname{dim} \pi^{K(m)}=(2 m-c(\pi)+1)(q-1) q^{r-1}+\sum_{i=r+1}^{m}(2(m-i)+1)(q-1)^{2} q^{i-2} .
$$

Proof. Note that $2 r \leq c(\pi)<2 r+2$. By Lemma 4.6 and (4.2), we get

$$
\begin{aligned}
\operatorname{dim} \pi^{K(m)} & =\sum_{i=0}^{m} \sum_{\lambda \in L_{i}}(2 m-c(\pi \otimes \lambda)+1) \\
& =\sum_{i=0}^{r} \sum_{\lambda \in L_{i}}(2 m-c(\pi)+1)+\sum_{i=r+1}^{m} \sum_{\lambda \in L_{i}}(2 m-2 c(\lambda)+1) \\
& =(2 m-c(\pi)+1)(q-1) q^{r-1}+\sum_{i=r+1}^{m}(2(m-i)+1)(q-1)^{2} q^{i-2},
\end{aligned}
$$

as required.

Corollary 4.8. Let $\pi$ be an irreducible minimal supercuspidal representation of $\mathrm{GL}_{2}(F)$. Set $r=\left\lfloor\frac{c(\pi)}{2}\right\rfloor$.

(i) If $c(\pi)$ is even, then $\operatorname{dim} \pi^{K(r)}=(q-1) q^{r-1}$.

(ii) If $c(\pi)$ is odd, then $\operatorname{dim} \pi^{K(r+1)}=(q+1)(q-1) q^{r-1}$.

In general, for an irreducible supercuspidal representation $\pi$ of $\mathrm{GL}_{2}(F)$, there exist an irreducible minimal supercuspidal representation $\tau$ of $\mathrm{GL}_{2}(F)$ and a quasi-character $\chi$ of $F^{\times}$such that $\pi=\tau \otimes \chi$. In fact, being supercuspidal is preserved under twisting by characters by the formula (9.5.2) of [4]. The existence of the minimal conductor among twists of $\pi$ guarantees. Thus, we can take a quasi character $\chi$ so that $c\left(\pi \otimes \chi^{-1}\right)=$ $\min \left\{c\left(\pi \otimes \chi^{\prime}\right) \mid \chi^{\prime}:\right.$ a quas-character of $\left.F^{\times}\right\}$. Then $\tau=\pi \otimes \chi^{-1}$ is an irreducible minimal supercuspidal representation which satisfies $\pi=\tau \otimes \chi$. 
Proposition 4.9. Let $\tau$ be an irreducible minimal supercuspidal representation of $\mathrm{GL}_{2}(F)$ and $\chi$ a quasi-character of $F^{\times}$. Set $\pi=\tau \otimes \chi$. For any integer $m$ such that $c(\pi) \leq 2 m$, we have

$$
\operatorname{dim} \pi^{K(m)}=\operatorname{dim} \tau^{K(m)}
$$

Proof. By (4.2), we see that $2 c(\chi) \leq c(\pi)$. So we have $c(\chi) \leq m$. Since the character $\chi \circ$ det of $\mathrm{GL}_{2}(F)$ is trivial on $K(m)$, the functor $\tau \mapsto \tau \otimes \chi$ preserves the $K(m)$-fixed vectors. This implies the assertion.

By Propositions 4.7 and 4.9, we obtain the following

Corollary 4.10. Let $\pi$ be an irreducible supercuspidal representation of $\operatorname{GL}_{2}(F)$. Set

$$
s=\min \left\{c(\pi \otimes \chi) \mid \chi: \text { a quasi-character of } F^{\times}\right\} \text {and } r=\left\lfloor\frac{s}{2}\right\rfloor .
$$

Then for any integer $m$ such that $c(\pi) \leq 2 m$, we have

$$
\operatorname{dim} \pi^{K(m)}=(2 m-s+1)(q-1) q^{r-1}+\sum_{i=r+1}^{m}(2(m-i)+1)(q-1)^{2} q^{i-2} .
$$

\section{REFERENCES}

[1] I. N. Bernšteĭn and A. V. Zelevinskiŭ, Representations of the group $G L(n, F)$, where $F$ is a local non-Archimedean field. Uspehi Mat. Nauk 31 (1976), no. 2 5-70.

[2] P. Broussous and B. Lemaire, Building of $G L(m, D)$ and centralizers. Transformation Groups 7 (2002), $15-50$.

[3] C. J. Bushnell, Hereditary orders, Gauss sums and supercuspidal representations of $G L_{N}$. J. Reine Angew. Math. 375/376 (1987), 184-210.

[4] C-J. Bushnell and G. Henniart, The local Langlands conjecture for GL(2). Grundlehren der Mathematischen Wissenschaften [Fundamental Principles of Mathematical Sciences], 335. Springer-Verlag, Berlin, 2006. xii +347 pp.

[5] W. Casselman, The restriction of a representation of $G L_{2}(k)$ to $G L 2(\mathfrak{o})$. Math. Ann. 206 (1973), 311-318.

[6] W. Casselman, Introduction to admissible representations of $p$-adic groups, available at his homepage.

[7] H. Jacquet, A correction to Conducteur des representations du groupe lineaire. Pacific J. Math. 260 (2012), no. 2, 515-525.

[8] H. Jacquet, I-I. Piatetski-Shapiro, and J. Shalika, Conducteur des representations du groupe lineaire. Math. Ann. 256 (1981), no. 2, 199-214. 
[9] H-H. Kim, S. Wakatsuki, and T. Yamauchi, Equidistribution theorems for holomorphic Siegel cusp forms of general degree: the level aspect, arXiv:2106.07811.

[10] J. Lansky and A. Raghuram, On the correspondence of representations between $G L(n)$ and division algebras, Proc. Amer. Math. Soc, 131 (2003), 1641-1648.

[11] N. Matringe, Essential Whittaker functions for GL(n). Doc. Math. 18 (2013), 1191-1214.

[12] A. Moy and G. Prasad, Unrefined minimal K-types for p-adic groups. Invent. Math. 116 (1994), no. 1-3, 393-408.

[13] D. Prasad and A. Raghuram, Representation theory of $G L(n)$ over non-Archimedean local fields. School on Automorphic Forms on $G L(n), 159-205$, ICTP Lect. Notes, 21, Abdus Salam Int. Cent. Theoret. Phys., Trieste, 2008.

[14] H. Saito, On Tunnell's formula for characters of $G L(2)$. Compositio Math. 85 (1993), no. 1, 99-108.

[15] J-A. Shalika, J. The multiplicity one theorem for $G L_{n}$. Ann. of Math. (2) 100 (1974), 171-193.

[16] S.W. Shin and N. Templier, Sato-Tate theorem for families and low-lying zeros of automorphic Lfunctions, Inv. Math., 203 (2016), no. 1, 1-177.

[17] J-B. Tunnell, On the local Langlands conjecture for $G L(2)$. Invent. Math. 46 (1978), no. 2, 179-200.

[18] H. Yoshida, On extraordinary representations of $G L_{2}$. Algebraic number theory (Kyoto Internat. Sympos., Res. Inst. Math. Sci., Univ. Kyoto, Kyoto, 1976), pp. 291-303. Japan Soc. Promotion Sci., Tokyo, 1977.

Michitaka Miyauchi, Graduate School of Education, Okayama Univ., 3-1-1, TsushimaNAKA, KiTA-KU, OKaYAma 700-8530 , JAPAN

Email address: miyauchi@okayama-u.ac.jp

Takuya Yamauchi, Mathematical Inst. Tohoku Univ., 6-3,Aoba, Aramaki, Aoba-Ku, SENDAI 980-8578, JAPAN

Email address: takuya.yamauchi.c3@tohoku.ac.jp 